\title{
THREE CASES OF FRACTURE OF THE FIBULA.
}

\author{
By E. H. BENNETT, M.D., F.R.C.S. ; \\ Surgeon to Sir P. Dun's Hospital; \\ Professor of Surgery, Trinity College.
}

[Read in the Section of Pathology, May 17, 1895.]

Wirtin the last two years I have had under my care in hospital three cases of injury of the ankle-joint, similar in kind and differing from each other only in the degree of dislocation of the joint, and in the time which elapsed in each case between the occurrence of the accidents which produced the dislocations and their presentation at hospital for treatment. The details of the fracture of the fibula which was present in each were exactly the same. The accidents that produced them were, respectively, a twist of the foot when alighting on the ground after jumping from a fence; tripping in a mowing machine by which the foot was fixed, while the body of the patient fell forwards over the machine; and lastly, a fall down a flight of stairs while carrying a heavy pail of water.

In all three the patients found themselves unable to use the limb immediately after the injury, and had to be carried to bed without attempting to put the injured limbs in use. It is not possible in accidents of this kind always to determine with exactness the quality of the wrench of the ankle which produced the fracture, but the inspection of a series of pathological specimens combined with clinical study, I think, removes all doubt as to their mechanism. I shall read the notes of only one of my cases in detail.

M. H. Q., aged fifty, was admitted to hospital on February 2nd, 1895, with a malunited fracture of the lower end of the left fibula. 
She gave the following account of her injury:-On October 31st, 1894, three months before her admission, while carrying a bucket of water downstairs, she slipped and fell down the whole flight twisting her foot some way-she does not remember howunder her. She made attempts to get up and walk, but found that she was quite unable to put her left foot to the ground owing to the intense pain caused by the effort as well as by the loss of power in the limb. She then called for help, and was carried up to bed by some neighbours who sent her next day to a hospital. On admission the limb, which to the best of her knowledge was without any deformity, was merely superficially handled and put up carelessly in a box splint packed around with some wadding. It was left so for six weeks without alteration or readjustment. When the box splint was removed, it was then loosely wrapped in a broad flannel bandage which was readjusted about every three weeks, until finally she left the hospital after three months from the date of the injury with the limb in a condition of most extreme deformity. [Cast here exhibited.]

This cast represents the condition of the ankle at this time The foot is in extreme extension, and is displaced backwards and outwards. A very deep sulcus separates the heel from the surface of the back of the leg, and on the inside the tibia with its malleolus projects strongly forwards and inwards. Over this projection the skin was tense and shining, and in part ulcerated. The foot and leg were odematous. The patient could make no attempt to use the limb further than to touch the ground with the great toe. In this case it is clear that a fracture was recognised as being present, else the box splint would not have been used. The woman states that nothing like the present deformity existed at that time. It is clear, I think, the deformity occurred during the lax treatment by the box splint.

It appears to me that we are not to attribute all the blame for these failures in diagnosis and treatment to the surgeons who have had the misfortune to maltreat them. The surgical writings of the present day which are accepted as text-books, contribute by their defects to errors of diagnosis, and so to 
had treatment. Many omit any description of the particular fracture which occurs in these cases-the fracture of the lower end of the fibula-caused by the tearing away of the external malleolus through the strain of the external lateral ligament of the ankle. For example:-

There is no mention of this injury in Erichsen or Pick's books, and the latter occupies himself only with the mechanism of Potts' fracture. If one takes as guides to the site of fractures of the lower end of the fibula from wrenching of the ankle, those who place the fracture from two to three inches from the lower end of the bone, the examples of the fracture before us will be readily overlooked; the more so if they be without displacement at the first. Equally erroneous is the statement that Potts' fracture is the commonest fracture of the lower end of the fibula. Erichsen writes:- "The fibula may be fractured about three inches above the ankle, the tip of the malleolus - that is, the inner malleolus-being splintered as well. This constitutes the form of injury called Yotts' fracture, and is, perhaps, the most common fracture in this situation."

As to treatment, too, we read strange directions. The newest American authority, who, indeed, recognises the existence of the injury, states that "No treatment is needed except rest, with the support of a dressing to prevent the occurrence of another twist of the foot." This looks like the treatment which has proved so disastrous in the case I have presented. If the relative frequency of this fracture is tested by grouping the specimens of the various fractures of the lower end of the fibula produced by indirect injury, contained in a large collection-such as the Museum of Trinity College contains-formed without any attempt at selection, we find that Potts' fracture, as strictly defined, does not occupy the position of the most common fracture.

If, then, one approaches the diagnosis and treatment of 
fractures of the lower end of the fibula without the knowled gre of the existence of this fracture, or, even recognising it, regards it as a rare and exceptional injury, errors of diagnosis and of treatment must follow. I can, in this Section, deal with treatment only so far as it helps to clear the pathology of the injury. In my three cases, manipulation combined with traction enabled me to break through the ill-placed callus, and to reduce the dislocation of the ankle in a great measure. The length of the time in the three cases was-in the first, a month; in the second, six weeks; in the last, three months.

Dr. Crory said that the fibula was not usually fractured as high "Ip as originally described hy Pott. If there is great tenderness over the lower end of the fibula when pressed on there is a fracture there. Very often it does not present the typical appearance of a Pott's fracture, the foot not being displaced. Pott never mentioned anything about the tip of the internal malleolus being knocked off. 